\title{
Atomic Force Microscopy of Structural-Mechanical Properties of Polyethylene Reinforced by Silicate Needle-Shaped Filler
}

\author{
Ilya A. Morozov, ${ }^{1,2}$ Oleg K. Garishin, ${ }^{1}$ Vladimir V. Shadrin, ${ }^{1,2}$ \\ Victor A. Gerasin, ${ }^{3}$ and Maria A. Guseva ${ }^{3}$ \\ ${ }^{1}$ Institute of Continuous Media Mechanics, UB RAS, 1 Akademika Koroleva Street, Perm 614013, Russia \\ ${ }^{2}$ Perm State University, 15 Bukireva Street, Perm 614990, Russia \\ ${ }^{3}$ Institute of Petrochemical Synthesis, RAS, 29 Leninsky Avenue, Moscow 119991, Russia
}

Correspondence should be addressed to Ilya A. Morozov; ilya.morozov@gmail.com

Received 14 September 2015; Accepted 20 December 2015

Academic Editor: Pavel Lejcek

Copyright (C) 2016 Ilya A. Morozov et al. This is an open access article distributed under the Creative Commons Attribution License, which permits unrestricted use, distribution, and reproduction in any medium, provided the original work is properly cited.

\begin{abstract}
The paper presents the results of experimental studies of polyethylene-based composites reinforced with silicate needle-shaped filler (palygorskite) of different mass fraction $(0,5,10$, and 15\%). These composites are less flammable and fire toxic than unfilled polyethylene. The structure (size, shape, and agglomeration of filler) and local mechanical properties of composites in nonstretched and elongated states were investigated by AFM. In stretched samples palygorskite takes a wavy shape, and at extremely high elongation the filler is orthogonal to the axis of tension. The smooth surfaces of the samples, required for AFM, were prepared using the heating/cooling procedure.
\end{abstract}

\section{Introduction}

Nanocomposites based on different polyolefins (polyethylene, polypropylene, etc.) and disperse silicate ultrafine particles (nanoclay) are currently the object of intense applied and fundamental investigations. This family of composite materials has some essential advantages, for instance, those associated with physical and mechanical properties, over conventional filled polymers. They have such valuable properties as improved performance, environmental friendliness, and relative cheapness and they are easy to manufacture. Polyolefins are the most common and available type of thermoplastic polymers.

Numerous studies [1-3] have shown that even small volume fractions of silicate particles strongly improve the diffusion barrier characteristics of polymers, their thermal stability, and buckling. This can most likely be attributed to the specific features of nanostructured materials. Unlike conventional materials (some components are of micron and submicron sizes), nanomaterials have very high surface/interface area, and therefore the fraction of interface layers formed on disperse particles can reach very high values. Modification of the physical properties of these fillers can improve essentially their macroproperties. An extremely small particle size hinders the separation of a matrix from inclusions due to the action of high surface tension forces, and this certainly increases the strength of the composite. All of these factors taken together contribute much to the effectiveness of one or another physical characteristic of the material at relatively low filler concentrations.

Beginning in 1974, ultrafine clay filler was incorporated into polyolefins [4]. The first commercial use of this material was in car interior elements. Also, it was widely used as packaging for liquid products. The high fire-resistance of the material in the first case and its excellent thermal insulation and antidiffusion properties in the second case drew the attention of manufacturers [5-8]. Currently, this material is finding ever increasing use in various industrial applications.

For the design of original nanocomposites with improved properties it is necessary to get knowledge of processes that take place inside the material at micro- and nanoscales and their effects on the mechanical behavior of material. 
In this paper, the results of complex experimental studies of polyethylene-based composites reinforced with silicate needle-shaped filler (palygorskite) are presented. The mechanical properties of these composites were analyzed. AFM in a nanomechanical mapping mode was used to perform the structural-mechanical analysis of samples, including those under elongation. Experiments were carried out with samples of relatively large sizes (necessary for stretching operation), and therefore the heating/cooling procedure to obtain the samples with smooth surfaces required for AFM measurements was used.

\section{Materials and Methods}

2.1. Preparation of Samples. The low density $\left(910 \mathrm{~kg} / \mathrm{m}^{3}\right)$ polyethylene PE107-02K, which has found wide application in industry, was studied. Differential scanning calorimetry (DSC) revealed that the degree of crystallinity of this material was about 33-35\%. The polymer was reinforced with palygorskite. This clay mineral is magnesium aluminum silicate, which has the approximate chemical formula $\left(\mathrm{Mg}_{2} \mathrm{Al}_{2}\right)\left(\mathrm{Si}_{8} \mathrm{O}_{20}\right)(\mathrm{OH})_{2}\left(\mathrm{OH}_{2}\right)_{4} \cdot 4 \mathrm{H}_{2} \mathrm{O}$ [9-11].

Palygorskite crystals are composed of double chains (bands) of silicon-oxygen tetrahedrons linked together by positive octahedrally coordinated magnesium and aluminum cations. At the grinding stage the mineral split into particles having the form of elongated columns. The density of palygorskite is $2000-2300 \mathrm{~kg} / \mathrm{m}^{3}$, and its Mohs hardness is from 2 to 2.5 [10]. Palygorskite minerals often contain a fraction of montmorillonite particles [11]. Since these minerals have similar chemical nature and density, it is difficult to separate them fully. Crystals of palygorskite and montmorillonite do not grow into each other and coexist in the mineral.

The surface of palygorskite crystals, like the surface of any other clay minerals, is hydrophilic, which hampers its moisture treatment by hydrophobic organic substances. So, it is necessary to treat the filler with surfactants (SAS) and create the organophilic layers on the clay surface which provide the required level of interaction with the polymer matrix. For surfactant, dimethyldioctadecylammonium bromide (DDAB) was used.

The composites were produced using one-stage melt mixing technology [12]. Polymer, silicate, and SAS were fed simultaneously into the twin-screw extruder Haake MiniLab Rheomex CTW5 (Thermo Electron Corporation). The Mini$\mathrm{Lab}$ is a microcompounder for blending of small amounts of material $\left(7 \mathrm{~cm}^{3}\right)$, equipped with conical screws $(5 / 14 \mathrm{~mm}$ in diameter and a length of $110 \mathrm{~mm}$ ), an integrated backflow channel, and an inert gas flush system. Temperature of mixing was chosen at $150^{\circ} \mathrm{C}$, which lies approximately in the middle between melting $\left(107^{\circ} \mathrm{C}\right)$ and beginning of thermal oxidative destruction of $\mathrm{PE}\left(190^{\circ} \mathrm{C}\right)$. It was established that under these conditions palygorskite particles were dispersed rather uniformly in the matrix and diffusion of SAS molecules onto the surface of silicate particles and modification took place directly during the blending. The filler fraction in the composites was $0,5,10$, and $15 \%$ of weight.
The surface of samples (work area $0.34 \times 4 \times 12 \mathrm{~mm}$ ) has many scratches and artificial defects. AFM examination requires proper preparation of the sample surface. To this end, a sample was placed into a furnace, where it was heated to the temperature close to its melting point $\left(130^{\circ} \mathrm{C}\right)$ and aged for $30 \mathrm{~min}$. After that, the temperature was reduced to $95^{\circ} \mathrm{C}$, and the material was left in the furnace for 2 hours more. As a consequence, crystallites were first melted and then recovered (recrystallized). A DSC analysis of materials before and after heating shows that the degree of crystallinity of samples upon heat treatment increases by $10 \%$. After such heating procedure, the surface became smoother and the natural characteristic features of the relief (crystallites, filler inclusions) became more pronounced. We studied not only the microstructure of nondeformed materials, but also the structure of large-sized stretched materials. Hence, there was a need to prepare the large smooth surface precisely by heat treatment, but not by cutting in a cryoultramicrotome which is able to prepare only a small area of the sample.

2.2. Mechanical Tests. The mechanical properties of composites at the macroscale were investigated at room temperature using a testing machine Testometric FS-100 CT. Tests were carried out with an elongation rate of $100 \% / \mathrm{min}(2$. $10^{-4} \mathrm{~m} / \mathrm{sec}$ ). Samples were stretched until complete failure. Five tests were performed for each set of samples.

2.3. Atomic Force Microscopy. Surface properties were studied using a Dimension Icon AFM in a nanomechanical mapping mode (PeakForce QNM). At each point of the surface relief the probe indents the sample with a frequency of $2 \mathrm{kHz}$. The approach and retraction force curves are processed in real time to give a map of the structural-mechanical properties of the surface: (1) height of the relief, (2) adhesion force, (3) depth of indentation of probe indentation into the material, and (4) stiffness of the material (elastic modulus $E$ calculated in terms of the Derjaguin-Muller-Toporov (DMT) model) [13]. The maps of adhesion, indentation, and stiffness give additional information about local inhomogeneities and mechanical properties.

The probes NSG10 (NT-MDT) with nominal radius $R=$ $10 \mathrm{~nm}$ and calibrated stiffness $\sim 9 \mathrm{~N} / \mathrm{m}$ were used. The applied force to the samples was set up to $5 \mathrm{nN}$.

A structural-mechanical analysis was performed for materials in a nondeformed state and those in a static stretched state. In the latter case samples were fixed in a small tensile device and placed directly under the microscope scanner.

Images of filled and unfilled materials are given in Figures 3 and 4 . For the publication space saving, the images were vertically cut out. The dimensions of images are $10 \times 10 \mu \mathrm{m}$ $(1024 \times 1024$ points in $x y$ plane $)$. Ten images were obtained and processed for each material. The length of the examined area is shown in the left corner of each image below the horizontal bar, and the ranges of the measured value and measurement units are given to the right of the vertical bar. 


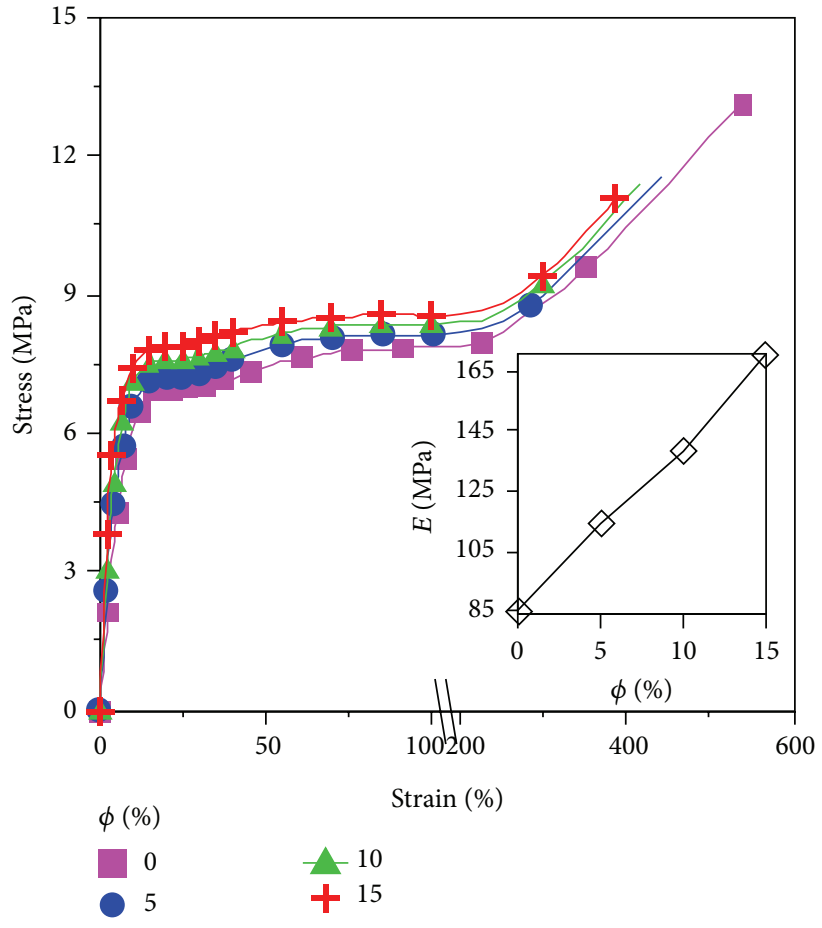

(a)

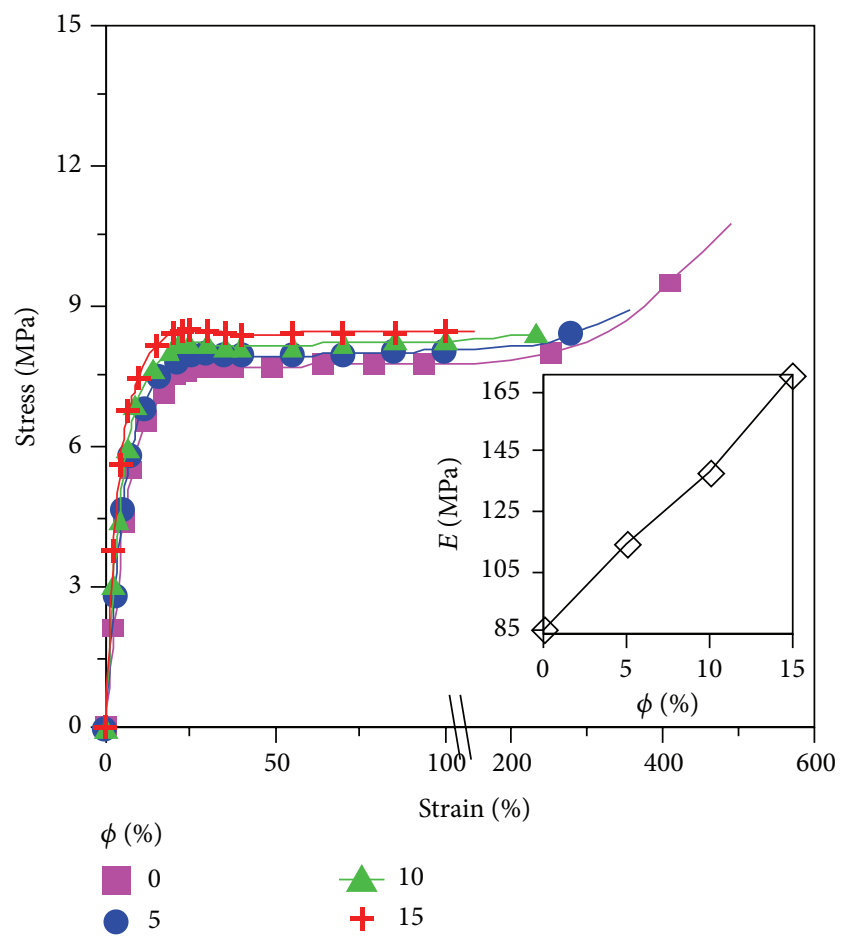

(b)

FIGURE 1: Stress-strain curves before (a) and after (b) thermal treatment. The relationships of initial elastic modulus versus $\phi$ are shown in inserts.

\section{Results and Discussion}

Figure 1 presents the nominal stress-strain curves obtained for samples before and after heat treatment. Experiments indicate that the mechanical behavior of original and thermally treated samples is nearly the same on the initial loading segment. Differences start at strain $\varepsilon>100-150 \%$. For thermally treated samples a slight decrease in stresses at the hardening stage is observed. It is interesting that at the stage of a plastic flow the curves $\sigma(\varepsilon)$ corresponding to different filler concentrations approach each other. Hence, it can be concluded that on the plastic loading the influence of filler on the stress is markedly weaker compared to the elastic loading segment.

The relationships between Young's modulus and the filler concentration are shown in inserts of Figure 1. The modulus linearly increases twice with increasing filler concentration, from $85 \mathrm{MPa}$ for unfilled polymer to $170 \mathrm{MPa}$ for $\phi=15 \%$.

Employing the Farris concentration relationship for convection polymeric composites and suspensions with grainy filler [14],

$$
E_{c}=E_{m}\left(1+\frac{1.78 \varphi_{v}}{\left(1-\varphi_{v} / 0.60\right)}\right)^{1.4},
$$

where $E_{c}$ is the composite modulus, $E_{m}$ is the matrix modulus, and $\varphi_{v}$ is the volumetric concentration. Bearing in mind that the filler density is twice as much as the matrix density, one can get $E_{c} \approx 1.5 E_{m}$. The experimental result is $E_{c} \approx 2.0 E_{m}$ (see inserts in Figure 1). Hence, the incorporation of given filler into the polymer provides better reinforcing of the material than that observed in conventional composites.

Mechanical tests have demonstrated that the heat treatment of samples based on the polyethylene under study has a slight effect on the elastic modulus and on the shape of loading curves. DSC studies revealed that the degree of crystallinity of the cured material did not change significantly; that is, the heat treatment did not have a strong effect on the internal structure of the material.

The AFM height and stiffness (elastic modulus) images of surface of unfilled polyethylene after heating and cooling are given in Figure 2.

As one can see, the unfilled polyethylene surface is relatively rough. This can be attributed to the inhomogeneous structure of the material (amorphous, crystalline areas) and the history of external effects on the sample surface. Nevertheless, the distribution of stiffness is rather homogeneous. The distribution histogram shown in Figure 3(a) corresponds to the stiffness map given in Figure 2(b). The mean value is $92 \mathrm{MPa}$, and this value only slightly exceeds the results of macroscopic tests $(85 \mathrm{MPa})$. The mean indentation depth of the unfilled material (histogram in Figure $3(\mathrm{~b})$ ) is $3.5 \mathrm{~nm}$ and the modeled value $2.3 \mathrm{~nm}$.

Figure 4 presents the maps of structural-mechanical properties of the surface of filled materials upon heat treatment. Filler inclusions are clearly seen on the indentation (dark areas, i.e., small depths of indentation) and stiffness (light areas, i.e., rigid surface fragments) maps. The polymer 


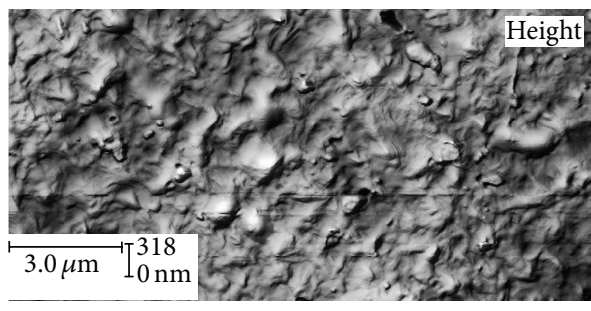

(a)

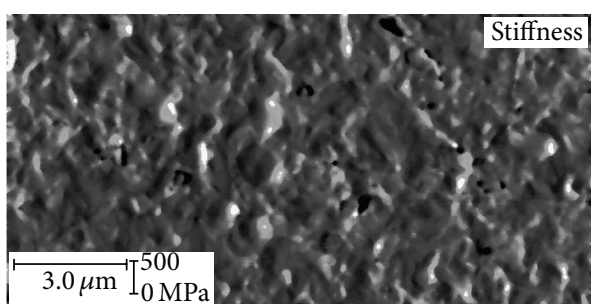

(b)

FIGURE 2: AFM images of surface height (a) and stiffness (b) of unfilled polyethylene.

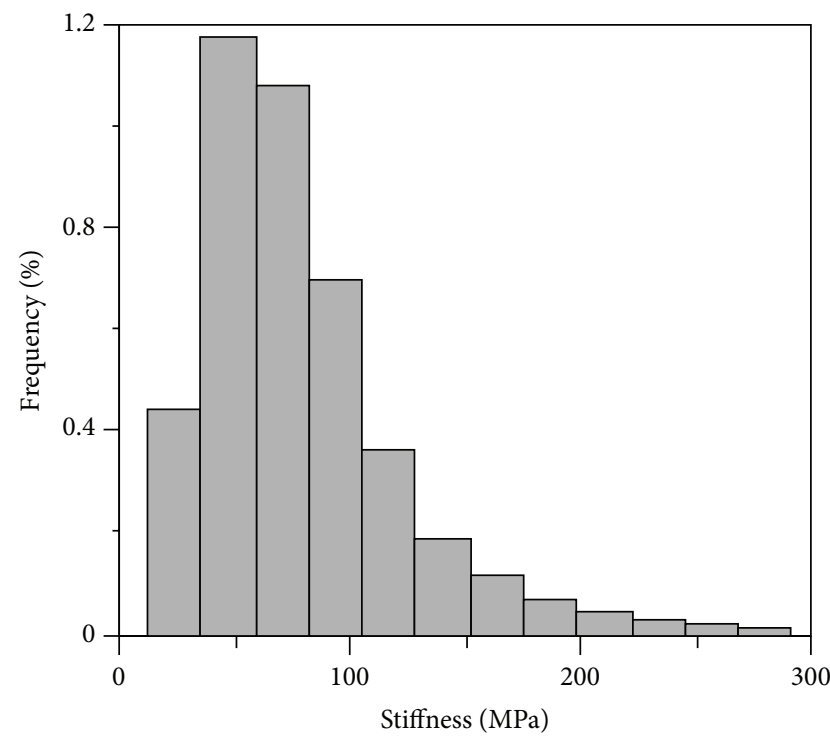

(a)

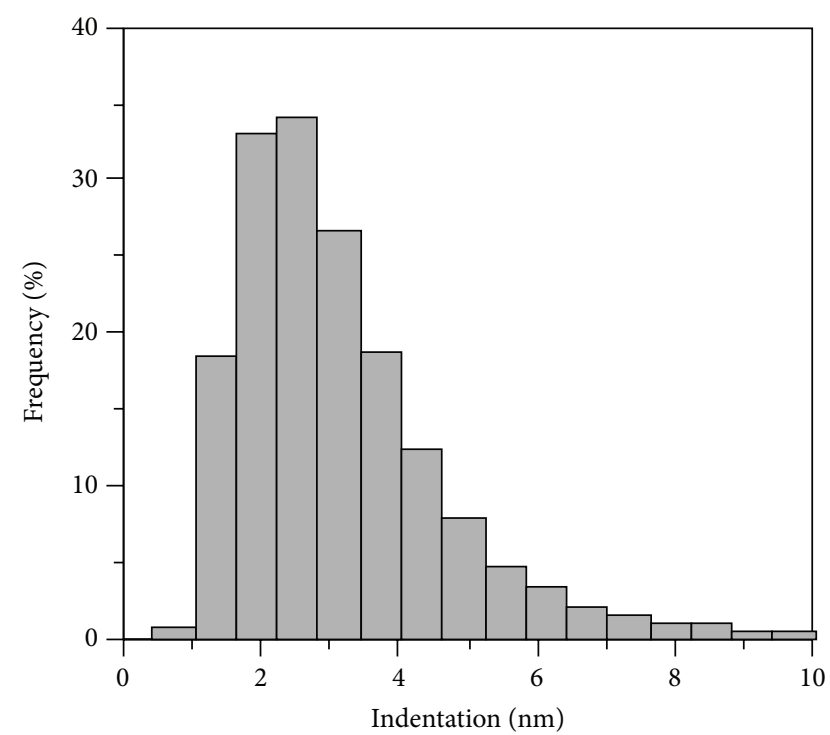

(b)

FIGURE 3: Distribution of stiffness (a) and indentation depth (b) for the unfilled polyethylene surface.

matrix corresponds to the light areas of indention (high indentation) and the dark ones, that is, low values of stiffness. The thread-like areas of the matrix correspond to lamella structure of polyethylene (Figure 4(a)).

Since the chosen AFM probes have cantilevers of relatively low stiffness, suitable for soft polymer materials, they cannot indent rigid filler inclusions. Hence, the shown upper stiffness limit of the material (1.5 GPa, Figure 4, right column) cannot be definitely correlated with the filler stiffness. This limit is a conditional value corresponding to an infinitely hard material.

Apart from needle-shaped filler (palygorskite), the flat inclusions of different sizes, which are shards of clay (montmorillonite), are also visible in the images (Figure 4(b)). Note that the area fraction of these flat large inclusions is comparable with the area occupied by palygorskite.

Despite the fact that filler inclusions can be readily found on the maps of mechanical properties, the polymer-filler interface has never sharp contrast; it always has a tilt of width of several (tens of) nanometers. This is coupled with the facts that some portion of filler particles can be hidden under the polymer surface and that the probe can slip out of the edges of inclusions. For the qualitative analysis of filler structure (in particular, palygorskite needle thickness), we need to work out the criteria for each point in the AFM image to elucidate whether it corresponds to polymer or to filler. To find a solution to this problem, the analysis of indentation depth maps is used.

Figure 5 presents the indentation distribution histograms corresponding to the maps in Figure 4. The material surface indentation map consists of the filler, polymer, and the transition zone indentation areas. At $\phi \geq 10 \%$, two distributions, one in the range of small indentation depth (peak $\approx 2 \mathrm{~nm}$ ) and the other in the area of relative deep indentation depth (peak $\approx 5-6 \mathrm{~nm}$ ), are seen in the histograms. With increasing filler fraction, the separation of the general histogram becomes more pronounced. The lack of peaks at $\phi=5 \%$ (Figure 5(a)) can be attributed to small fraction of rigid phase.

The same pattern can be seen in all examined images. Assuming that filler indentation proceeds within the interval $\leq 2.3 \mathrm{~nm}$, parts that correspond to a given area of the images are retained. Figure 6 demonstrates filler silhouettes specified in Figure 5.

Hence, the two-color black and white images were obtained and treated with structural analysis. A total of several hundred needle-shaped inclusions acquired from 

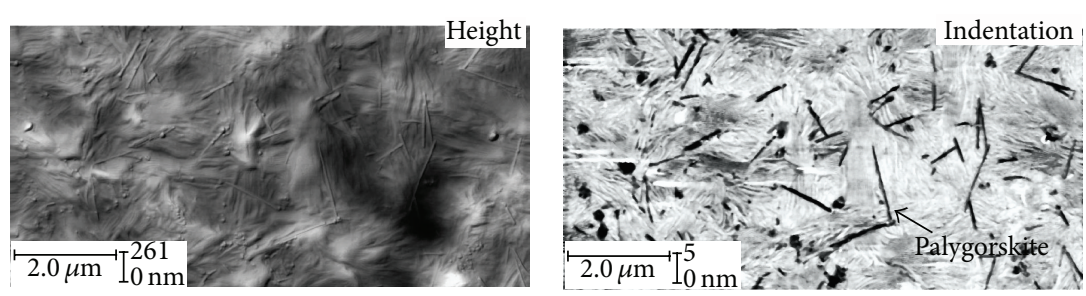

(a)
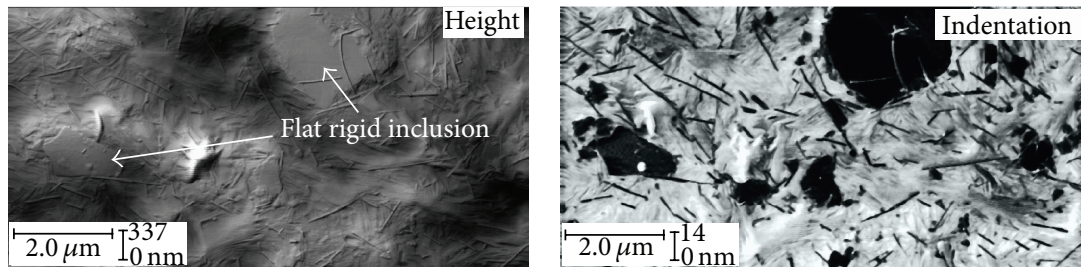

(b)
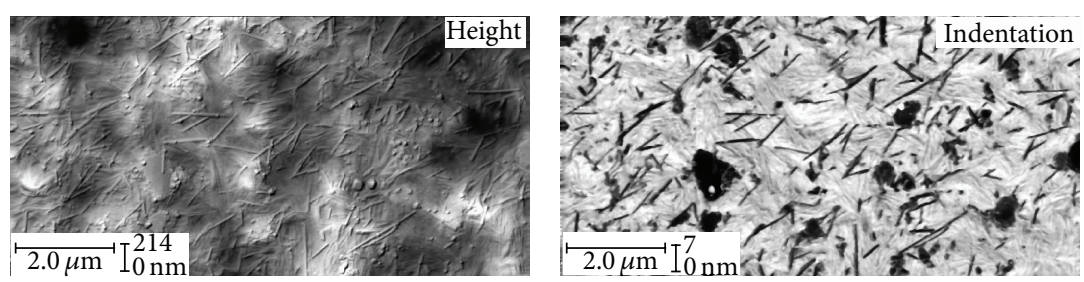

(c)
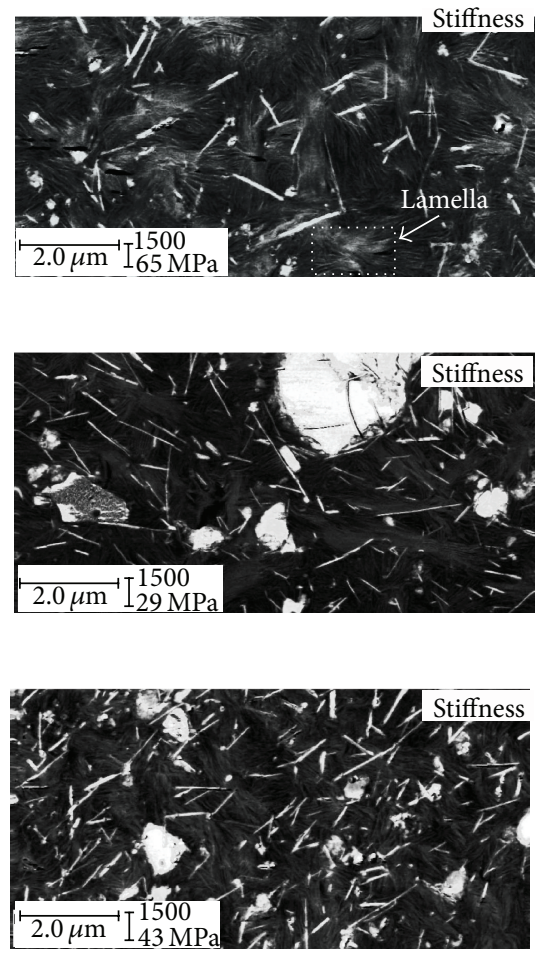

FIGURE 4: AFM images of surface height (left column), indentation depth (middle column), and stiffness (right column): (a) $\phi=5 \%$, (b) $\phi=$ $10 \%$, and (c) $\phi=15 \%$.

images for each material were analyzed. Figures 7(a) and 7(b) give palygorskite thickness and length distributions.

The measured mean thickness of needles (Figure 7(a)) is $20-30 \mathrm{~nm}$, and their mean length (Figure $7(\mathrm{~b})$ ) is $0.5 \mu \mathrm{m}$. Small fraction of long needles up to $1.8 \mu \mathrm{m}$ is also observed.

It is found that palygorskite forms in the material secondary structures, several inclusions arranged parallel and closely to one another (Figure 8). Therefore, accurate identification of the boundaries of individual needles is not always possible, and hence the length and thickness of the measured objects can be overestimated. This explains the occurrence of the second thickness distribution peak for $\phi$ $=5 \%$ (in the range $60 \mathrm{~nm}$ ) and the shift of the curve for $\phi=$ $15 \%$ to the right (Figure $7(\mathrm{a})$ ).

The surface structure of the material $(\phi=10 \%)$ extended twice is given in Figure 9; the axis of elongation is vertical. The results indicate that part of needles takes a wavy shape. Apparently, this can be attributed to the inhomogeneous local deformation of polyethylene and/or to the compression of the material in a direction orthogonal to elongation.

It has also been found that the polymer separates at the poles of large-size inclusions of montmorillonite. Figure 10 illustrates two various regions with separations caused by 50\% (Figure 10(a)) and 100\% (Figure 10(b)) elongation.

As stretching increases, the material surface becomes significantly rough; that is, the structure of oriented bumps and cavities occurs, and effects of palygorskite bending and montmorillonite separation increase. The roughness of the surface also increases the errors of nanomechanical mapping and accurate measurement of stiffness is not always possible. Hence, as material-contrast value, in Figure 9 the adhesion, which is less sensitive to the roughness, was shown.

In the state of extreme elongation, the tip of open notch in the stretched material (Figure 11(a)), the polyethylene forms strands that join the edges of rupture (Figures 11(b) and 11(c)).

Filler (clearly seen on the stiffness map, Figure 11(c)) is oriented together with strands and perpendicular to the elongation (rupture) axis.

\section{Conclusions}

The structure and mechanical properties of polyethylenebased composites reinforced with silicate needle-shaped filler (palygorskite) were investigated. The advantage of such composites over unfilled polyethylene is that they are less flammable and fire toxic. The results of mechanical tests show that the initial modulus increases approximately twice due to presence of filler.

For preparation of samples for AFM the heating/cooling procedure was used, which made it possible to obtain largesized samples with smooth surfaces.

The microstructural analysis indicates that apart from needle-shaped filler (palygorskite) there are also single 


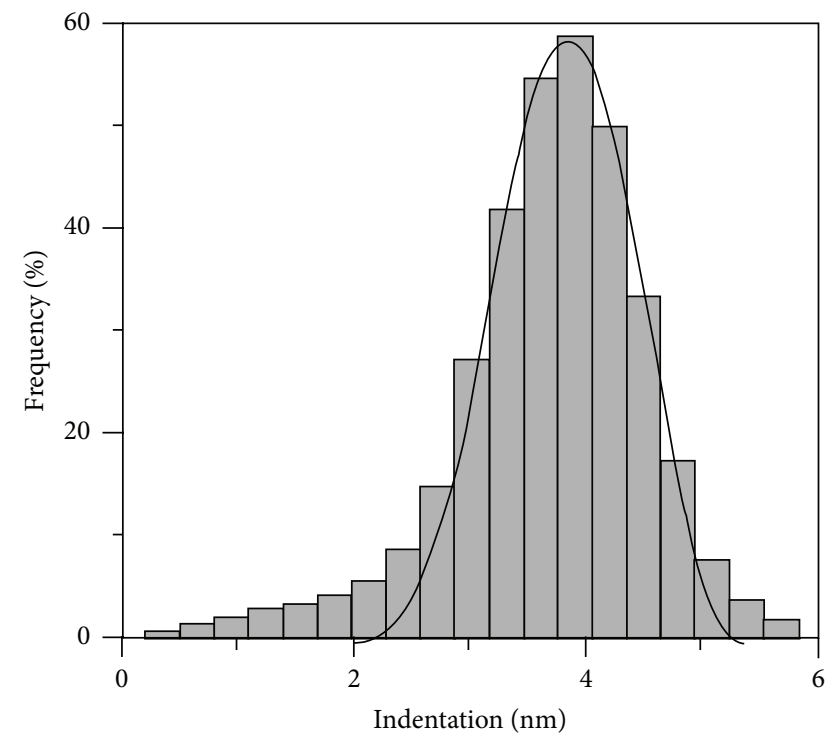

(a)

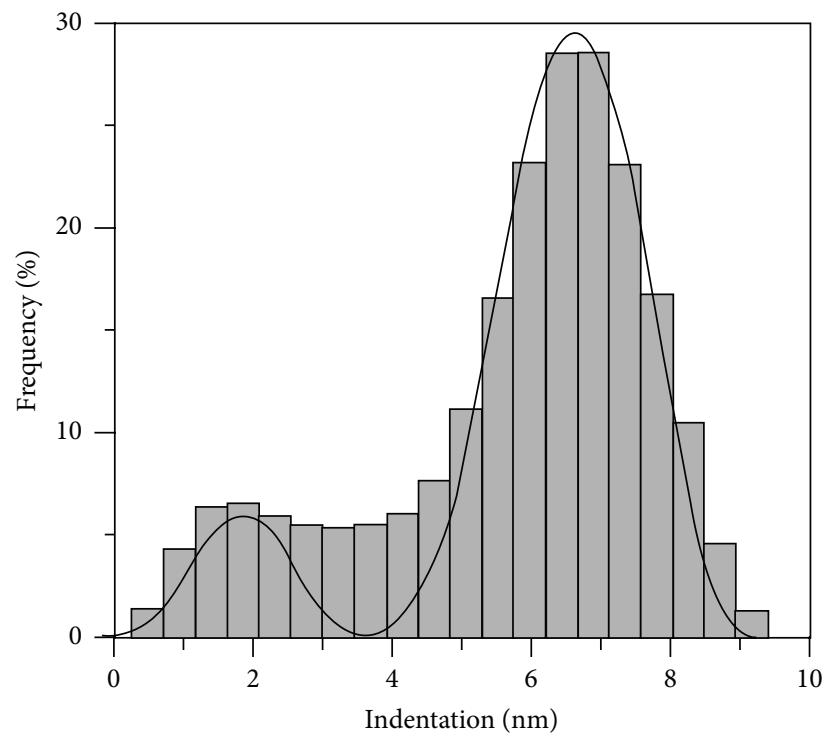

(b)

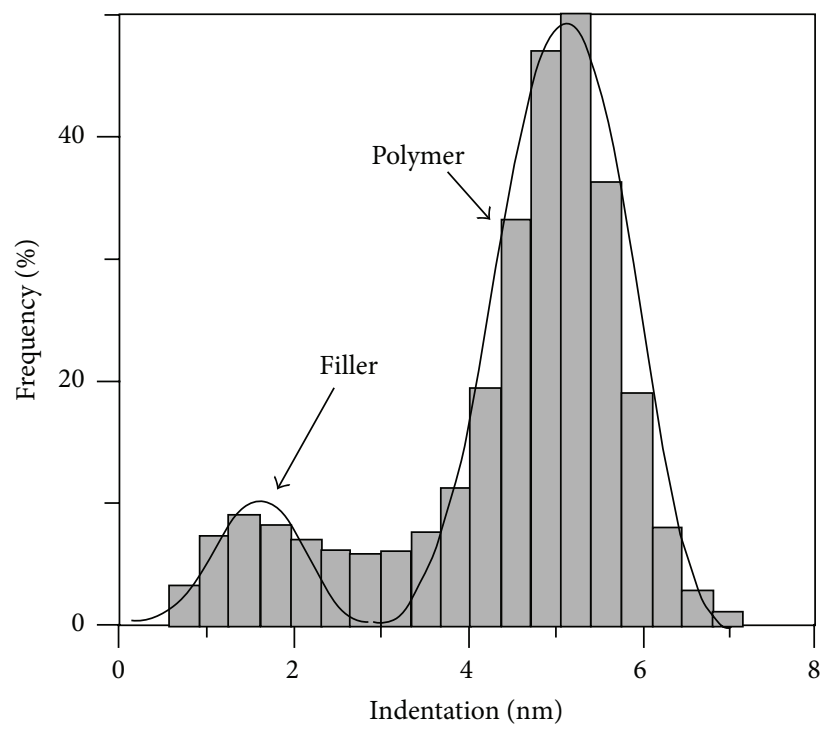

(c)

FIGURE 5: Indentation depth distribution histograms for the materials: (a) $\phi=5 \%$, (b) $\phi=10 \%$, and (c) $\phi=15 \%$.

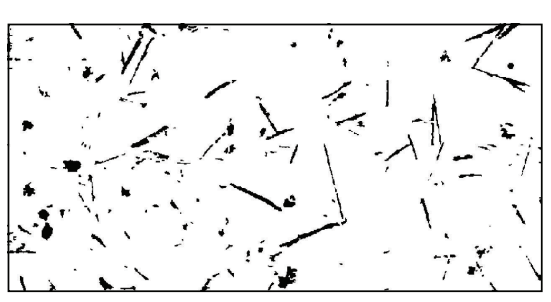

(a)

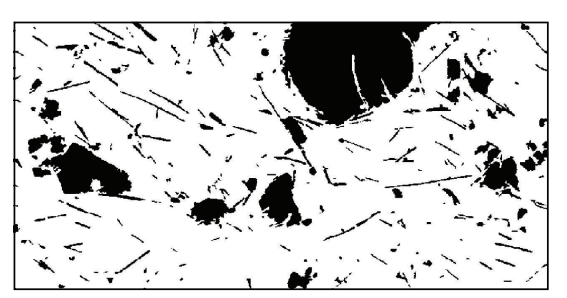

(b)

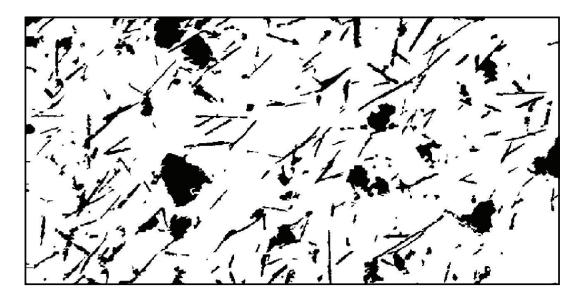

(c)

Figure 6: Filler silhouettes (see Figure 4): (a) $\phi=5 \%$, (b) $\phi=10 \%$, and (c) $\phi=15 \%$. 


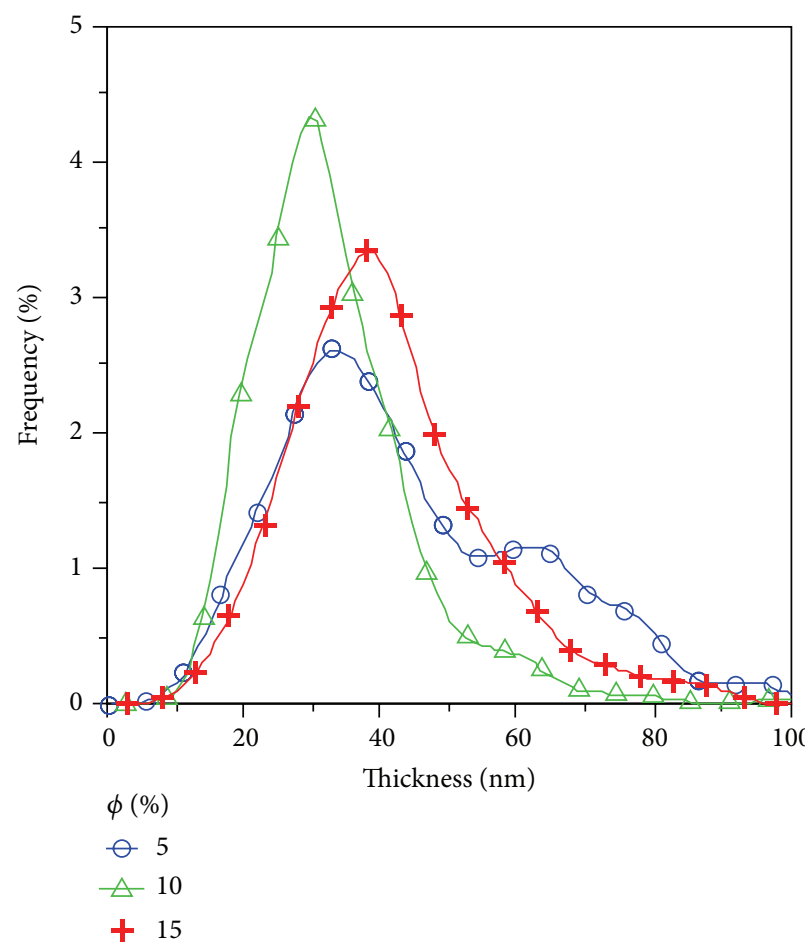

(a)

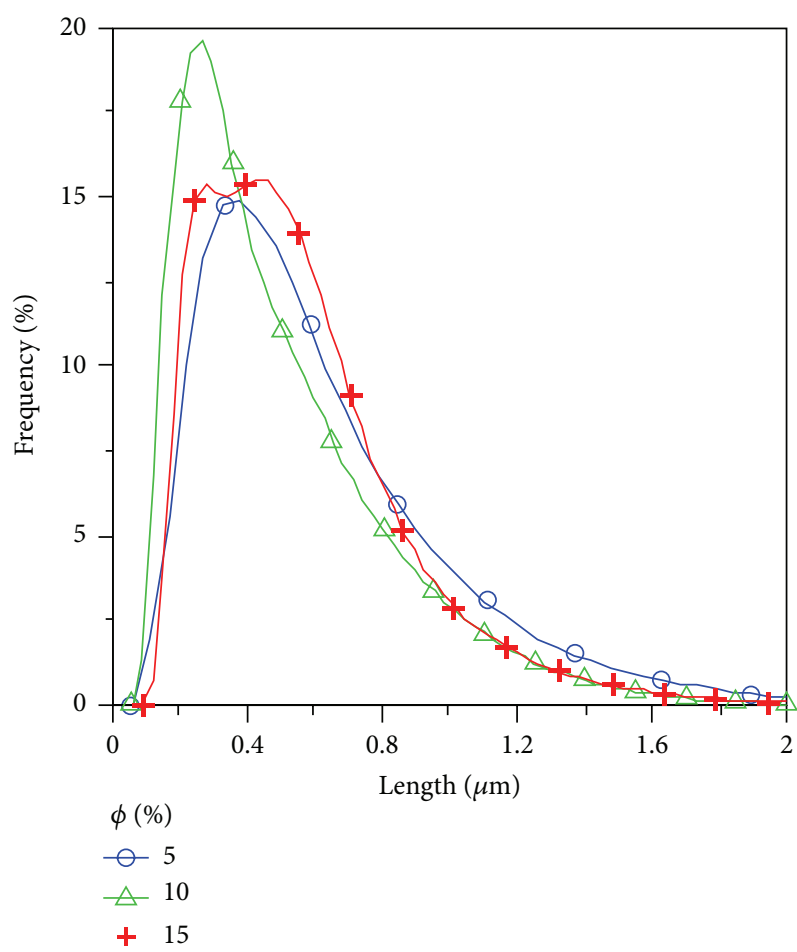

(b)

FIGURE 7: Distributions of thickness (a) and length (b) of palygorskite.

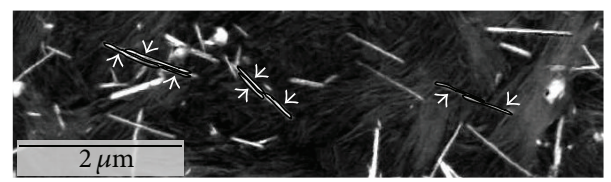

FIGURE 8: AFM stiffness map for the material $\phi=10 \%$. Secondary structures formed from neighboring parallel needles are marked out with black lines. The lamella structure of polyethylene is visible as grey filaments.
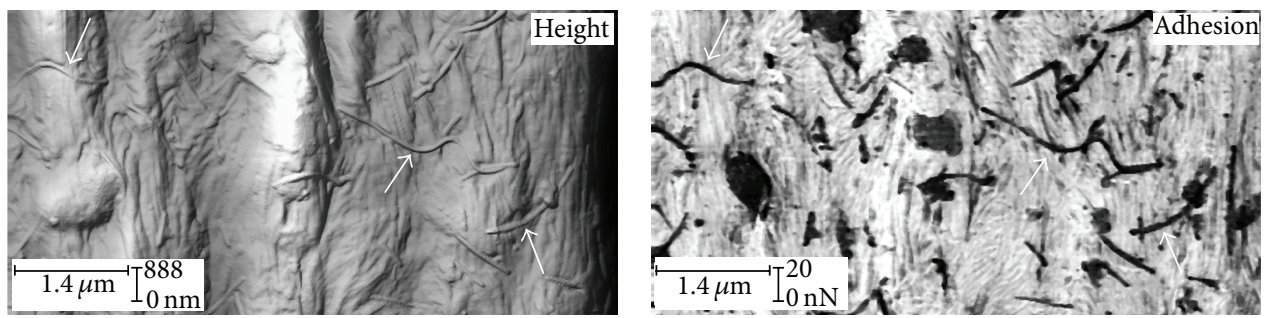

Figure 9: AFM height and its surface adhesion map for the material with $\phi=10 \%$ at $100 \%$ elongation. Arrows denote the wavy structure of palygorskite.

flat inclusions of different sizes, which are the clay particles (montmorillonite). The study of the indentation maps allowed us to determine the polymer-filler boundary and to estimate the sizes of palygorskite inclusions: the mean thickness of needles $20-30 \mathrm{~nm}$ and their mean length $0.5 \mu \mathrm{m}$. There are also long needles whose length reaches $1.8 \mu \mathrm{m}$. The agglomeration of palygorskite into secondary structures was observed. Therefore, accurate identification of the boundaries of individual needles is not always possible.

It has been found that the microstructure of palygorskite in stretched composite takes a wavy shape, and the matrix separates from the flat micron-sized inclusions of clay. In the case of extreme elongation (the tip of the open notch in the stretched material), the polyethylene forms strands that 


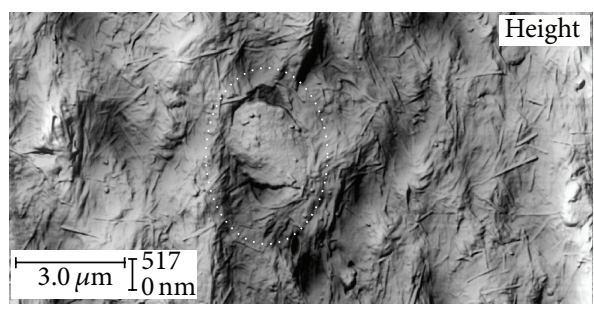

(a)

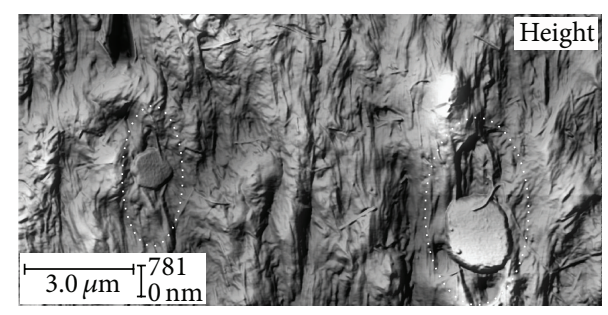

(b)

Figure 10: Polymer separation at the poles of flat inclusions. Elongation of the material is $50 \%$ (a) and $100 \%$ (b).

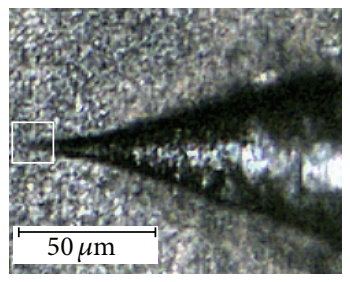

(a)

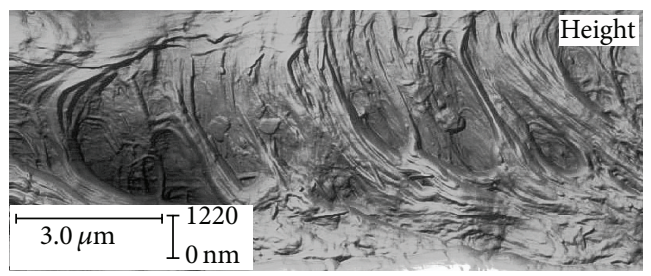

(b)

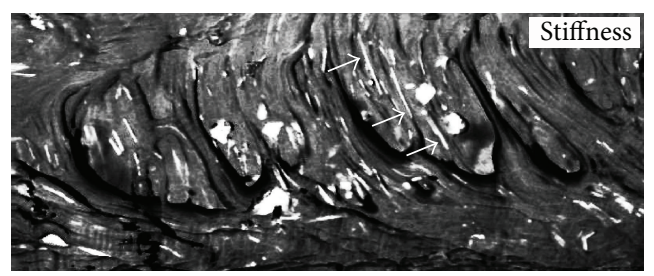

(c)

FIGURE 11: Optical image of the open notch (a) and AFM image of the relief height (b) and stiffness (c) of the material at the notch tip of the stretched material.

connect the edges of rupture. In this case, palygorskite in the strands is oriented together with them perpendicular to the rupture axis.

\section{Conflict of Interests}

The authors declare that there is no conflict of interests regarding the publication of this paper.

\section{Acknowledgments}

This work is supported by RFBR: Grants 13-08-00065 (macroexperiment), 14-01-96002 r_urals_a (AFM experiment), and 15-03-08483 (fabrication of nanocomposite specimens), and UB RAS Program 15-10-1-18.

\section{References}

[1] F. Gao, "Clay/polymer composites: the story," Materials Today, vol. 7, no. 11, pp. 50-55, 2004.

[2] N. Sheng, M. C. Boyce, D. M. Parks, G. C. Rutledge, J. I. Abes, and R. E. Cohen, "Multiscale micromechanical modeling of polymer/clay nano-composites and the effective clay particle," Polymer, vol. 45, no. 2, pp. 487-506, 2004.

[3] M. Manitiu, R. J. Bellair, S. Horsch, E. Gulari, and R. M. Kannan, "Supercritical carbon dioxide-processed dispersed polystyreneclay nanocomposites," Macromolecules, vol. 41, no. 21, pp. 80388046, 2008.

[4] P. D. Shepherd, F. J. Golemba, and F. W. Maine, "Clay layers," Advances in Chemistry Series, vol. 41, pp. 134-141, 1974.

[5] J. W. Gilman, M. R. Nyden, and C. L. Jackson, New Approaches to the Development of Fire-Safe Materials, US Department of Commerce, Technology Administration, National Institute of Standards and Technology, Gaithersburg, Md, USA, 2000.
[6] J. W. Gilman, "Flammability and thermal stability studies of polymer layered-silicate (clay) nanocomposites," Applied Clay Science, vol. 15, no. 1-2, pp. 31-49, 1999.

[7] J. W. Gilman, T. Kashiwagi, M. R. Nyden et al., "Flammability studies of polymer layered silicate nanocomposites: polyolefin, epoxy, and vinyl ester resins," in Chemistry and Technology of Polymer Additives, chapter 14, pp. 249-265, Blackwell Science, Malden, Mass, USA, 1999.

[8] F. Dietsche and R. Mülhaupt, “Thermal properties and flammability of acrylic nanocomposites based upon organophilic layered silicates," Polymer Bulletin, vol. 43, no. 4, pp. 395-402, 1999.

[9] Z. Du, W. Zhang, C. Zhang, Z. Jing, and H. Li, "A novel polyethylene/palygorskite nanocomposite prepared via in-situ coordinated polymerization," Polymer Bulletin, vol. 49, no. 2-3, pp. 151-158, 2002.

[10] G. D. Chryssikos, V. V. Gionis, G. H. Kacandes et al., "Octahedral cation distribution in palygorskite," American Mineralogist, vol. 94, no. 1, pp. 200-203, 2009.

[11] V. V. Nasedkin, N. M. Boeva, I. A. Garbuzova, M. V. Kovalchuk, and A. L. Vasiliev, "The crystal structure and chemistry of several palygorskite samples with different geneses," Crystallography Reports, vol. 54, no. 5, pp. 884-900, 2009.

[12] M. Alexandre, G. Beyer, C. Henrist et al., “'One-pot’ preparation of polymer/clay nanocomposites starting from $\mathrm{Na}^{+}$montmorillonite. 1. Melt intercalation of ethylene-vinyl acetate copolymer," Chemistry of Materials, vol. 13, no. 11, pp. 3830-3832, 2001.

[13] B. V. Derjaguin, V. M. Muller, and Y. P. Toporov, "Effect of contact deformations on the adhesion of particles," Journal of Colloid and Interface Science, vol. 53, no. 2, pp. 314-326, 1975.

[14] R. J. Farris, "Prediction of the viscosity of multimodal suspensions from unimodal viscosity data," Journal of Rheology, vol. 12, no. 2, pp. 281-301, 1968. 

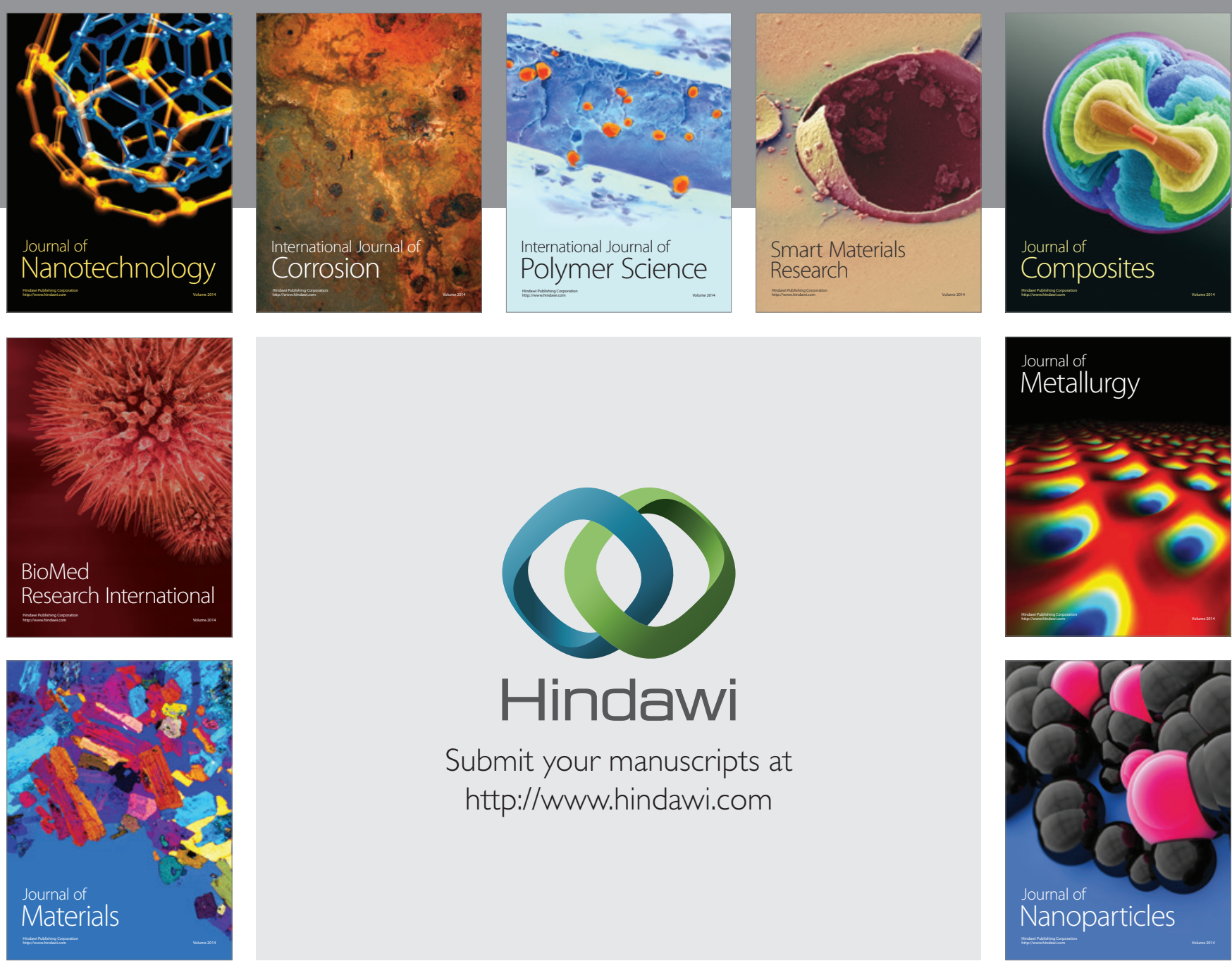

\section{Hindawi}

Submit your manuscripts at

http://www.hindawi.com

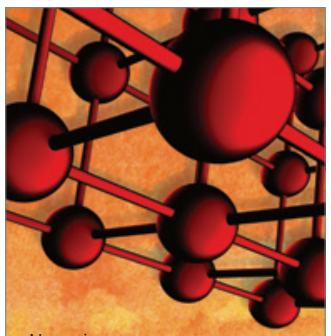

Materials Science and Engineering
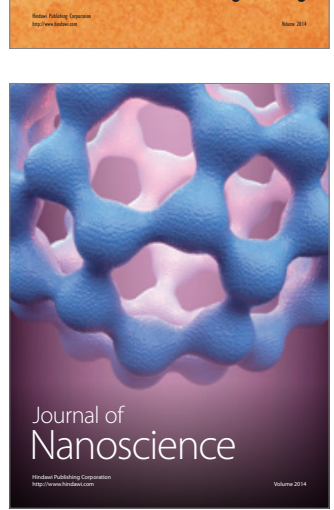
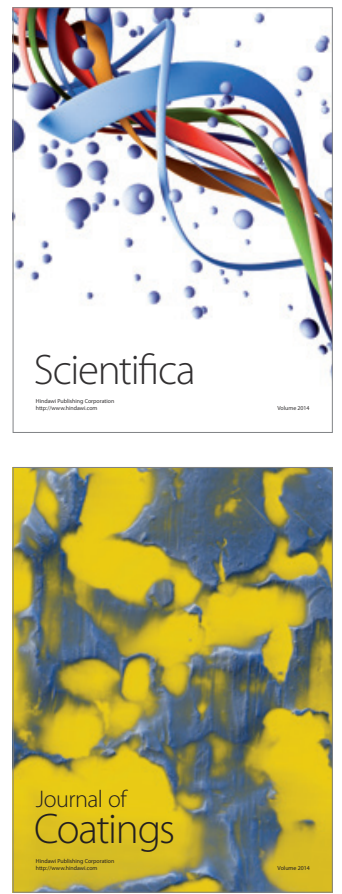
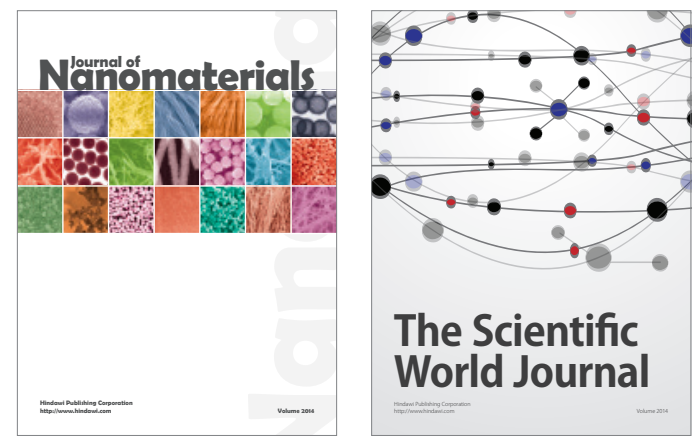

The Scientific World Journal
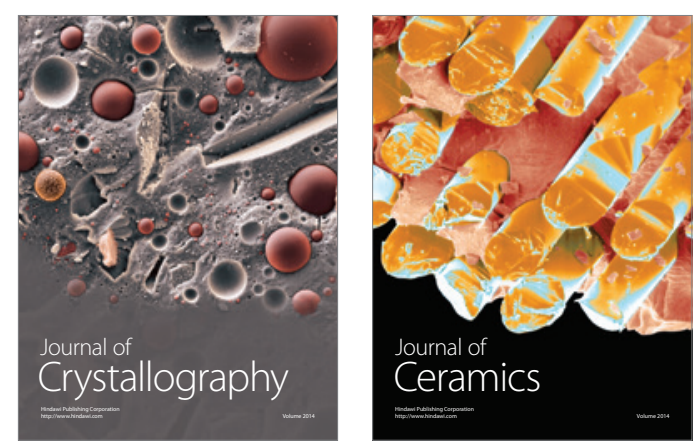
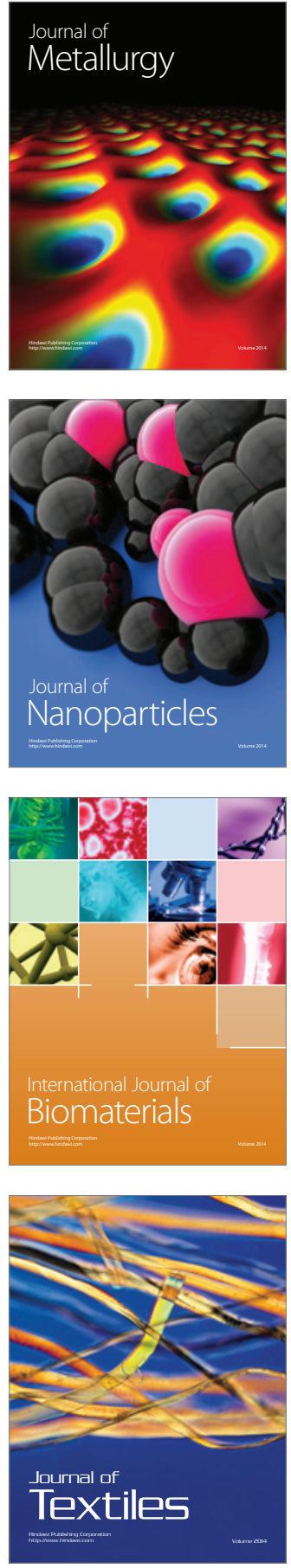